\title{
Power consumption rate analysis of Bioloid humanoid robot: towards energy saving and source development
}

\begin{abstract}
Specific energy (watt-hour/kg), which represents the density of battery energy, is the most important factor in a biped humanoid robot because it acts on all robot component functions. The energy source and robot load power consumption rate of this factor were analyzed in detail. A detailed analysis based on energy equation and experimental measurements was conducted on the energy consumption for 18 servo-actuators humanoid robot. The objective of such analysis was to identify and calculate the power consumption rate of each robot element during task motion time and running time of the robot and to study the effects of the energy drop on the controller and sensors. The findings of this study would provide insight into energy consumption analysis and motion planning tasks in a biped robot. The analysis was implemented on both individual and multi servos of motion tasks using a Bioloid premium (type A). The capture of energy consumption is analyzed and discussed in terms of robot dynamics during motion or interaction with sensors and control approaches to achieve a desired dynamic behavior. Modern technology instrument capabilities and fundamentals of energy calculations were adopted to obtain reliable and accurate measurements for two modes of operations, reference power source, and onboard battery source, which were compared and considered in the development of a new onboard energy source.
\end{abstract}

Keyword: Biped humanoid robot motion planning; Humanoid actuators; Servo motor energy calculation; Battery energy conservation 